\title{
Antenna Optimization Through Space Mapping
}

\author{
Jiang Zhu, John W. Bandler, Life Fellow, IEEE, Natalia K. Nikolova, Senior Member, IEEE, and \\ Slawomir Koziel, Member, IEEE
}

\begin{abstract}
We apply space mapping to antenna design for the first time. We exploit a coarse-mesh method of moments (MoM) solver as the coarse model and align it with the fine-mesh MoM solution through space mapping. We employ two plans: (I) implicit and output space mapping, and (II) input and output space mapping. We propose a local meshing method which avoids inconsistencies in the coarse model. The proposed techniques are implemented through our user-friendly space mapping framework (SMF) system. In a double annular ring antenna example, the S-parameter is optimized. The finite ground size effect for the MoM is efficiently solved by space mapping plan I and the design specification is satisfied after only three iterations. In a patch antenna example, we optimize the impedance using both plans in separate optimization processes. Comparisons are made. Coarseness in the coarse model and its effect on the space mapping performance are also discussed.
\end{abstract}

Index Terms-Antenna design, CAD, EM optimization, method of moments (MoM), space mapping (SM).

\section{INTRODUCTION}

$\mathbf{T}$ HE method of moments (MoM) is one of the most often used numerical techniques for antenna and microwave device analysis. Accurate MoM simulations of practical structures are memory and CPU intensive. This computational cost could become prohibitive in complex design problems, which may require anywhere between tens and thousands of system analyses. The electromagnetics-based antenna design remains a challenging task and ongoing research is pursuing acceleration of the numerical analysis on the one hand and improving the efficiency of the optimization algorithms on the other. The space mapping approach proposed here addresses the latter problem in conjunction with MoM-based analysis.

Common classifications of optimization algorithms include gradient-based (e.g., steepest-descent, conjugate-gradient, Newton and quasi-Newton), stochastic (e.g., random-search, genetic, simulated-annealing, particle-swarm), and neural-based

Manuscript received February 27, 2006; revised September 11, 2006. This work was supported in part by the Natural Sciences and Engineering Research Council of Canada under Grants OGP0007239 and STGP269760, and in part by Bandler Corporation.

J. Zhu, N. K. Nikolova, and S. Koziel are with the Simulation Optimization Systems Research Laboratory and the Computational Electromagnetics Research Laboratory, Department of Electrical and Computer Engineering, McMaster University, Hamilton, ON L8S 4K1, Canada.

J. W. Bandler is with the Simulation Optimization Systems Research Laboratory and the Computational Electromagnetics Research Laboratory, Department of Electrical and Computer Engineering, McMaster University, Hamilton, ON L8S 4K1, Canada and also with Bandler Corporation, Dundas, ON L9H 5E7, Canada.

Color versions of one or more of the figures in this paper are available online at http://ieeexplore.ieee.org.

Digital Object Identifier 10.1109/TAP.2007.891544 approaches [1]. Some of these have already been incorporated into commercial electromagnetic CAD packages. Only recently, the space mapping (SM) and surrogate-based optimization have been recognized in the mathematical and engineering community as a distinct class of approaches, e.g., [2]-[5]. They promise unprecedented efficiency in problems where two system models are available: a coarse model, which is not accurate but is fast (e.g., approximate analytical models, empirical formulas, equivalent circuits), and a fine model, which is very accurate but is expensive to evaluate (e.g., electromagnetic simulations, a set of measurements). The SM technique takes advantage of the efficiency of the coarse model and the accuracy of the fine model. It aligns the coarse model with the fine model in an iterative optimization process where most of the burden of the multiple system analyses is placed on the coarse model [6]-[10].

So far, SM has been successfully applied to the electromagnetics-based design of microwave filters, impedance-matching networks, multiplexers, etc.; see, e.g., [6]-[10]. There, equivalent-circuit, empirical, and semianalytical models and combinations thereof have been linked to full-wave electromagnetic simulators.

The application of SM to antenna design, on the other hand, proves to be more challenging. This is due to the fact that, with few exceptions, the radiating structures are too complex to lend themselves to analytical and/or circuit modeling. Examples of exceptions include printed patches of standard shapes and some standard horn designs. In the former case, fast analytical models include transmission-line and cavity representations loaded with the aperture admittances [11], [12]. In the latter case, complete design procedures exist [13] based on analysis where the edge diffraction is ignored. Such fast models are good candidates for coarse models in an SM optimization process. Unfortunately, for the vast majority of modern antenna types, coarse models are not available. This limits the applicability of the SM and surrogate-based optimization in antenna design.

Here, we propose an approach, which allows the construction of a coarse model for any radiating structure, which can be modeled by an MoM solver. The same solver is used for the coarse and the fine model. The fine model is an accurate MoM solution whose mesh satisfies rigorous convergence criteria, e.g., the convergence error is below $1 \%$. The coarse model uses a coarse mesh, which normally would satisfy very relaxed requirements, e.g., a convergence error below $15 \%$. Some MoM analysis engines may have additional features allowing for other types of approximations, which can speed up the system analysis significantly. A good example is the use of a specialized Green's function in the case of a ground plane and an $N$-layered substrate of infinite extent ( $N$ is finite), which is much faster than 
the analysis of a structure with a finite-size ground plane where all interfaces are meshed.

When employing coarse-mesh coarse models, the mesh topology must remain unchanged throughout the optimization iterations in order to keep the coarse-model response smooth and consistent in the design-parameter space. For that, local mesh control is employed. This is not necessary for the fine model, which can be remeshed at each optimization iteration.

We implement two SM plans: (I) implicit and output SM, and (II) input and output SM, [14], [15]. The aim of implicit and input SM is to roughly align the responses of the coarse and fine models through parameter extraction in the coarse-model design-parameter space. Then output SM performs responselevel adjustments to achieve a perfect local match between the coarse and fine model responses.

The optimization is carried out with the space mapping framework (SMF) system [16]. SMF is a prototype GUI-oriented software package that implements a number of SM optimization algorithms. The system provides sockets to popular simulators (e.g., MEFiSTo, FEKO, Sonnet em, ADS). It allows for automatic fine and coarse model data acquisition and, consequently, for fully automatic SM optimization. SMF also provides interfaces for modeling and statistical analysis.

Our examples are implemented with a commercial MoM solver [17]. We consider a double annular ring antenna and a patch antenna. In the first example, we exploit the CPU-intensive surface equivalence principle as a fine model and the special Green's function with a coarse mesh as a coarse model. The $S$-parameter response is optimized in three iterations. In the second example, we optimize the input impedance at a single frequency using two SM plans. The comparisons show larger time saving in SM plan I. Last, we discuss the coarseness in the coarse model and its effect on the SM performance.

\section{BASICS OF SPACE MAPPING OPtIMIZATION}

Space mapping (SM) technology is a recognized engineering optimization paradigm, consisting of a number of efficient optimization approaches, e.g., [5]-[10]. The main feature is that the direct optimization of the high-fidelity computationally expensive fine model is replaced by the iterative optimization and update of the fast coarse model. Provided that the misalignment between the fine and coarse models is not significant, SM-based algorithms typically provide excellent results after only a few evaluations of the fine model. In contrast, direct optimization typically requires dozens or hundreds of evaluations and may fail to provide acceptable results.

Let $R_{f}: X_{f} \rightarrow R^{m}$ denote the response vector of the fine model of a given device, where $X_{f} \subseteq R^{n}$ is the design-parameter domain of the fine model. Our goal is to solve

$$
\boldsymbol{x}_{f}^{*}=\arg \min _{x \in X_{f}} U\left(\boldsymbol{R}_{f}(\boldsymbol{x})\right)
$$

where $U: R^{m} \rightarrow R$ is a given objective function and $x$ is the vector of fine-model design parameters. In many engineering problems, we are concerned with the so-called minimax objective function. If we denote the fine model response components by $\boldsymbol{R}_{f}=\left[R_{f .1} \cdots R_{f . m}\right]^{T}$, the lower specifica- tion vector by $\boldsymbol{R}_{l}=\left[R_{l .1} \cdots R_{l . m}\right]^{T}$, and the upper specification vector by $\boldsymbol{R}_{u}=\left[R_{u .1} \cdots R_{u . m}\right]^{T}$, then we require that $R_{f . i} \leq R_{u . i}$ for $I \in I_{u}$ and $R_{f . i} \geq R_{l . i}$ for $I \in I_{l}$, where $I_{l}$, $I_{u} \subset\{1,2, \ldots, m\}$. The minimax objective function is defined as

$$
U\left(\boldsymbol{R}_{f}\right)=\max \left\{\max _{i \in I_{u}}\left(R_{f . i}-R_{u . i}\right), \max _{i \in I_{l}}\left(R_{l . i}-R_{f . i}\right)\right\} .
$$

In some problems, $U$ may be defined by a norm, i.e.

$$
U\left(\boldsymbol{R}_{f}\right)=\left\|\boldsymbol{R}_{f}-\boldsymbol{R}_{\text {spec }}\right\|
$$

where $\boldsymbol{R}_{\text {spec }}=\left[R_{\text {spec. } 1} \cdots R_{\text {spec.m }}\right]^{T}$ is the target response.

We consider the fine model to be expensive to compute and solving (1) by direct optimization to be impractical. Instead, we use surrogate models, i.e., computationally cheap models that are supposed to be acceptable local representations of the fine model.

According to the SM approach, the basis of the surrogate is the coarse model. Let $\boldsymbol{R}_{c}: X_{c} \times X_{p} \rightarrow R^{m}$ denote the response vectors of the coarse model. Here, $X_{c} \subseteq R^{n}$ is the coarse-model design-parameter domain (we assume that $X_{c} \subseteq X_{f}$ ) and $X_{p} \subseteq$ $R_{q}$ is the domain of auxiliary (preassigned) coarse model parameters. We emphasize that the preassigned parameters $\boldsymbol{x}_{p}$ are outside of $X_{f}$ and $X_{c}$. They are used to align the coarse and fine model responses in the parameter-extraction step of the SM procedure; however, they remain fixed during the optimization process aiming at an optimum surrogate with respect to the design specifications. Typical preassigned parameters in a layered structure are the dielectric constant and the height of a dielectric layer.

The optimal solution of the coarse model $\boldsymbol{x}_{c}^{*}$ is

$$
\boldsymbol{x}_{c}^{*} \triangleq \arg \min _{\boldsymbol{x} \in X_{c}} U\left(\boldsymbol{R}_{c}\left(\boldsymbol{x}, \boldsymbol{x}_{p}^{(0)}\right)\right)
$$

where $\boldsymbol{x}_{p}^{(0)}$ denotes the initial preassigned parameter values. Solving (4) is a necessary step in initializing the SM optimization process.

We consider an optimization algorithm that generates a sequence of points $\boldsymbol{x}_{f}^{(i)} \in X_{f}, i=1,2, \ldots$, so that

$$
\boldsymbol{x}_{f}^{(i+1)}=\underset{\boldsymbol{x} \in X_{s}^{(i)}}{\arg \min } U\left(\boldsymbol{R}_{s}^{(i)}(\boldsymbol{x})\right) .
$$

Here, $R_{s}^{(i)}: X_{s}^{(i)} \rightarrow R^{m}$ is the surrogate model at iteration $i$. We assume that $X_{f} \cap X_{s}^{(i)} \neq \varnothing$ for $i=0,1,2, \ldots$ In the SM framework, the family of surrogate models is constructed from the coarse model in such a way that each $\boldsymbol{R}_{s}^{(i)}$ is a suitable distortion of $\boldsymbol{R}_{c}$, such that the response of the surrogate model matches the response of the fine model as well as possible.

In this work, we use a surrogate model based on input SM [6], implicit SM [7] and output SM [18]. The surrogate model at iteration $i$ is defined as

$$
\boldsymbol{R}_{s}^{(i)}(\boldsymbol{x})=\boldsymbol{R}_{c}\left(\boldsymbol{B}^{(i)} \boldsymbol{x}+c^{(i)}, \boldsymbol{x}_{p}^{(i)}\right)+\Delta \boldsymbol{R}^{(i)}
$$




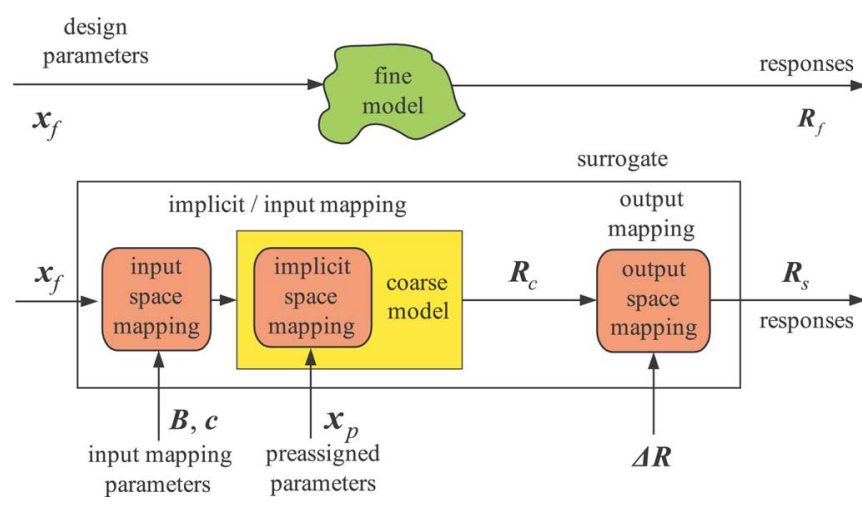

Fig. 1. Illustration of our approach to implicit, input, and output SM.

where

$$
\left(\boldsymbol{B}^{(i)}, \boldsymbol{c}^{(i)}, \boldsymbol{x}_{p}^{(i)}\right)=\arg \min _{\left(\boldsymbol{B}, \boldsymbol{c}, \boldsymbol{x}_{p}\right)} \varepsilon^{(i)}\left(\boldsymbol{B}, \boldsymbol{c}, \boldsymbol{x}_{p}\right)
$$

and

$$
\Delta \boldsymbol{R}^{(i)}=\boldsymbol{R}_{f}^{(i)}\left(\boldsymbol{x}_{f}^{(i)}\right)-\boldsymbol{R}_{c}\left(B^{(i)} \boldsymbol{x}_{f}^{(i)}+\boldsymbol{c}^{(i)}, \boldsymbol{x}_{p}^{(i)}\right) .
$$

The matrices $\boldsymbol{B}^{(i)} \in M_{n \times n}, \boldsymbol{c}^{(i)} \in M_{n \times 1}$, and the vector $\boldsymbol{x}_{p}^{(i)}$ are obtained using parameter extraction applied to the matching condition $\varepsilon^{(i)}$ as per (7). The vector $\Delta \boldsymbol{R}^{(i)} \in M_{m \times 1}$ is calculated using (8) after $\boldsymbol{B}^{(i)}, \boldsymbol{c}^{(i)}$, and $\boldsymbol{x}_{p}^{(i)}$ are determined.

A matching condition that we use in this work is defined as

$$
\varepsilon^{(i)}\left(B, c, x_{p}\right)=\left\|\boldsymbol{R}_{f}\left(\boldsymbol{x}_{f}^{(i)}\right)-\boldsymbol{R}_{c}\left(\boldsymbol{B} \cdot \boldsymbol{x}_{f}^{(i)}+\boldsymbol{c}, \boldsymbol{x}_{p}\right)\right\| .
$$

As follows from (7) and (9), we tune the values of the preassigned parameters $\boldsymbol{x}_{p}^{(i)}$ of the coarse model (implicit SM) and/or $\boldsymbol{B}$ and $\boldsymbol{c}$ (linear input SM) in order to reduce the misalignment between the fine model and the current surrogate model using the fine model data from the latest iteration. More general matching conditions can be found in [19].

It is now clear that implicit and input SM exploit the physicsbased similarity of the models and tune their shape, material or equivalent-circuit parameters, while the output SM ensures perfect local alignment between their responses at the current iteration point. As follows from (6)-(9), we implement implicit/input SM and output SM sequentially. This is illustrated in Fig. 1.

Having defined the surrogate model, we can define the optimization algorithm. It is, in fact, an implementation of the generic surrogate model-based optimization problem (5).

Step 1) Choose coarse model and preassigned parameters $x_{p}$. Set $i=0$.

Step 2) Solve (4) to find coarse-model optimal solution $\boldsymbol{x}_{c}^{*}$; set $\boldsymbol{x}_{f}^{(0)}=\boldsymbol{x}_{c}^{*}$.

Step 3) Evaluate fine model to obtain $\boldsymbol{R}_{f}\left(\boldsymbol{x}_{f}^{(i)}\right)$.

Step 4) Update $\boldsymbol{B}^{(i)}, \boldsymbol{c}^{(i)}, \boldsymbol{x}_{p}^{(i)}$ through parameter extraction using (7).

Step 5) Compute $\Delta \boldsymbol{R}^{(i)}$ using (8) and update surrogate model using (6).
Step 6) Solve (5) to obtain $x_{f}^{(i+1)}$.

Step 7) If termination condition is satisfied (convergence achieved or design specification satisfied), stop; otherwise, set $i=i+1$, go to Step 3 .

\section{COARSE-MESh COARSE MODELS}

In the numerical examples discussed in Section $\mathrm{V}$, both fine and coarse models are implemented using the same MoM-based simulator [17]. The fine model uses a fine mesh satisfying mesh convergence so that the results are accurate. The fine model evaluation is computationally demanding. The coarse model uses a coarse MoM mesh which, as indicated here, results in significant speed-up at the expense of the response accuracy.

\section{A. CPU Time Cost Versus Mesh Density}

The CPU time for an MoM simulation can be expressed as [20]

$$
T_{\mathrm{CPU}}=A+B N+C N^{2}+D N^{3}
$$

where $N$ is the number of unknowns. $A, B, C$, and $D$ are constants independent of $N$. $A$ accounts for the simulation setup. The meshing of the structure leads to the linear term $B N$. The filling of the system matrix is responsible for the quadratic term, and solving the matrix equation for the cubic term. The values of $A, B, C$ and $D$ depend on the problem at hand and the type of the MoM discretization procedure.

The quadratic and cubic terms dominate. For small to medium size problems, as the constant $C$ is much larger than $D$, the solution time is dominated by the matrix fill. For large-scale problems, the matrix solving time with its cubic term will eventually dominate the CPU-time cost. Thus, for medium to large scale problems, the time saving offered by coarse-mesh coarse models will be significant.

\section{B. Mesh Convergence and Meshing Method}

The mesh convergence needs to be checked to get an accurate simulation result. This is done by refining the mesh from one simulation to the next, and keeping all other parameters the same. If the results are significantly different, the surfaces are not adequately discretized and we need to refine the mesh.

The coarse-mesh coarse model does not need to achieve mesh convergence. Consequently, if the mesh topology and number of mesh elements vary due to the variation of geometrical design parameters during optimization, inconsistent results are obtained. To overcome this problem, we force the mesh topology to remain unchanged during the optimization. This is done by local meshing in FEKO [21]. In effect, for a given part of the structure, the user can fix the number of mesh lines along its contours. This enforces the same mesh topology regardless of variations in some of the shape parameters of the part.

In the fine model, where mesh convergence is satisfied, we use global meshing where the minimum mesh density is determined by the number of mesh lines per wavelength. At each iteration, remeshing is allowed. 


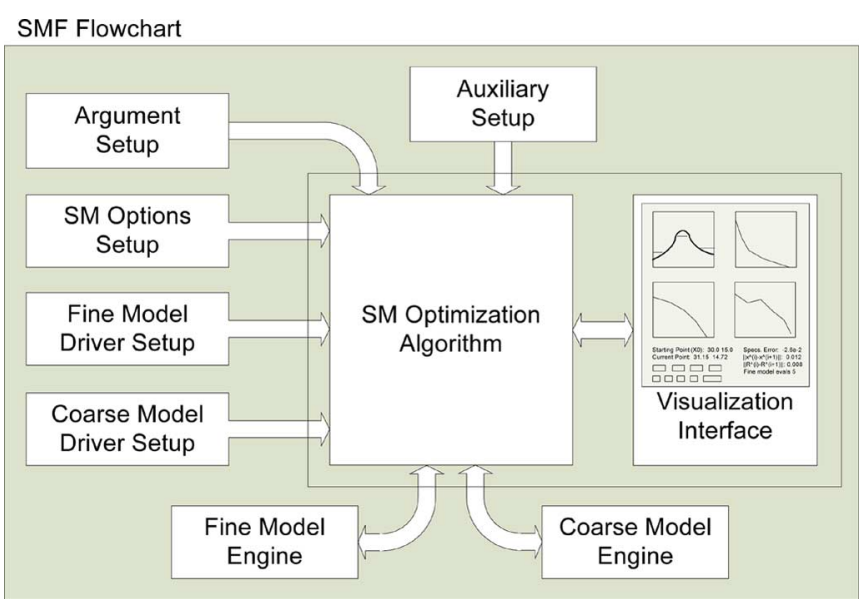

Fig. 2. Flowchart of the optimization module in SMF [16].

\section{SMF: IMPLEMENTATION OF SM OPTIMIZATION ALGORITHMS}

In order to make SM accessible to engineers not experienced in this technology, a prototype user-oriented software package was created. SMF [16] is a GUI-based Matlab system that can perform SM-based constrained optimization, modeling and statistical analysis. It implements existing SM approaches, including input, output, implicit and frequency SM. It contains drivers for commercial simulators that allow linking the fine and coarse model to the algorithm and make the optimization process fully automatic. In this paper, we use SMF to validate the antenna design based on SM optimization and MoM coarse and fine models.

Fig. 2 shows a block diagram of the optimization module in SMF. Optimization is performed in several steps. First, the user enters problem arguments, including starting point, frequency sweep, optimization type and specifications. Next, the user sets up space mapping itself, i.e., the kind of space mapping to be used (e.g., input, output, implicit), specifies the termination condition, parameter extraction options, and optional constrains.

The next step is to link the fine and coarse models to SMF by setting up the data that will be used to create model drivers. Using the user-provided data (e.g., simulator input files and design-parameter identification data), SMF creates drivers that automatically invoke fine and coarse model evaluations as required by the SM algorithm.

Parameter extraction, surrogate model optimization, and optional trust-region specific options are set in the next step using auxiliary interfaces.

Having done the setup, the user runs the execution interface, which invokes the SM optimization algorithm and the output visualization. The latter includes model responses, specification error plots as well as convergence plots, all updated at each SM iteration.

\section{EXAMPLES}

We use: (I) implicit and output SM, or (II) input and output SM. As explained in Section III, the implicit SM operates on the

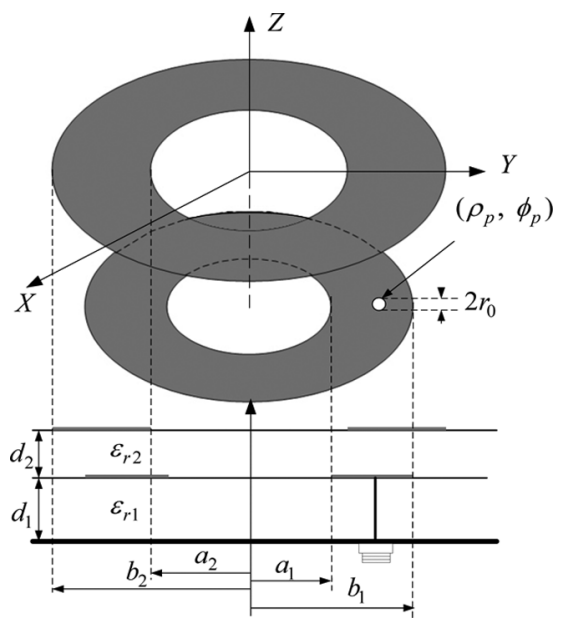

Fig. 3. Geometry of a stacked probe-fed printed double annular ring antenna example.

preassigned parameters while the input SM operates on the mapping parameters $\boldsymbol{B}$ and $\boldsymbol{c}$. Both approaches can be used separately (as done in the examples below) or simultaneously. When implicit SM is used alone, the input mapping parameters are fixed at $\boldsymbol{B}=\boldsymbol{I}$ and $\boldsymbol{c}=\mathbf{0}$, where $\boldsymbol{I}$ is the identity matrix. When input SM is used alone, $\boldsymbol{x}_{p}$ is empty.

\section{A. Double Annular Ring Antenna}

We consider the stacked probe-fed printed annular ring antenna [22] shown in Fig. 3. The antenna is printed on a printed circuit board (PCB) with $\varepsilon_{r 1}=2.2, d_{1}=6.096 \mathrm{~mm}$ for the lower substrate, and $\varepsilon_{r 2}=1.07, d_{2}=8.0 \mathrm{~mm}$ for the upper substrate. The dielectric loss tangent is 0.001 for both layers.

The finite ground size is $100 \times 100 \mathrm{~mm}$. The radius of the feed pin is $r_{0}=0.325 \mathrm{~mm}$. The design parameters are the outer and inner radius of each ring and the feed position, namely, $\left[\begin{array}{lllll}a_{1} & a_{2} & b_{1} & b_{2} & \rho_{p}\end{array}\right]^{T}$. The design specification is

$$
\left|S_{11}\right| \leq-10 \mathrm{~dB} \text { for } 1.75 \mathrm{GHz} \leq \omega \leq 2.15 \mathrm{GHz} .
$$

In the MoM solver used here, special Green's functions are available to model multilayer substrates where the ground plane and the substrate are assumed infinite in extent. The method is efficient since only the finite metallic surfaces are discretized. However, in many applications, the infinite ground plane assumption is not accurate. It is well known that the ground-plane size has a strong effect on the performance of microstrip antennas [23], [24].

The surface equivalence principle (SEP) allows the analysis of layered structures with a finite-size ground plane. In this case, however, the surfaces of all dielectric interfaces are discretized for the electric and magnetic current densities. All sides of a dielectric have to be meshed, making a closed solid. Thus, the number of unknowns is many times larger in comparison with the analogous structure of infinite ground plane analyzed with the special Green's function.

We choose the SEP model as the fine model and the special Green's function analysis as the coarse model. To further reduce the simulation time in the coarse model, we apply a coarser mesh 


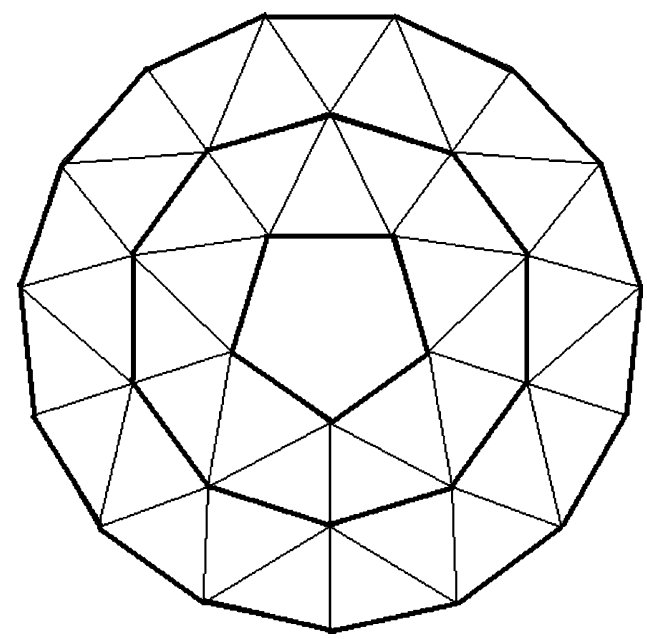

Fig. 4. Demonstration of local meshing of the annular ring in the coarse-mesh coarse model for a stacked ring antenna example.

TABLE I

Fine Model and CoARse Model in the Double AnNular Ring ANTEnNA

\begin{tabular}{ccccc}
\hline $\begin{array}{c}\text { Model } \\
\text { type }\end{array}$ & Technique & Meshing method & $\begin{array}{c}\text { Mesh } \\
\text { number }\end{array}$ & $\begin{array}{c}\text { Frequency } \\
\text { sweep time }\end{array}$ \\
\hline $\begin{array}{c}\text { Coarse } \\
\text { model }\end{array}$ & $\begin{array}{c}\text { Special Green's } \\
\text { function }+ \\
\text { coarse mesh }\end{array}$ & Local meshing & 83 & 8.721 seconds \\
\hline $\begin{array}{c}\text { Fine } \\
\text { model }\end{array}$ & SEP & $\begin{array}{c}\text { Global meshing } \\
\text { density }=20\end{array}$ & $2661^{*}$ & $\begin{array}{c}1 \text { hour and } 18 \\
\text { minutes }\end{array}$ \\
\hline \hline
\end{tabular}

* Number of mesh lines and time cost in the fine model are measured at the initial point.

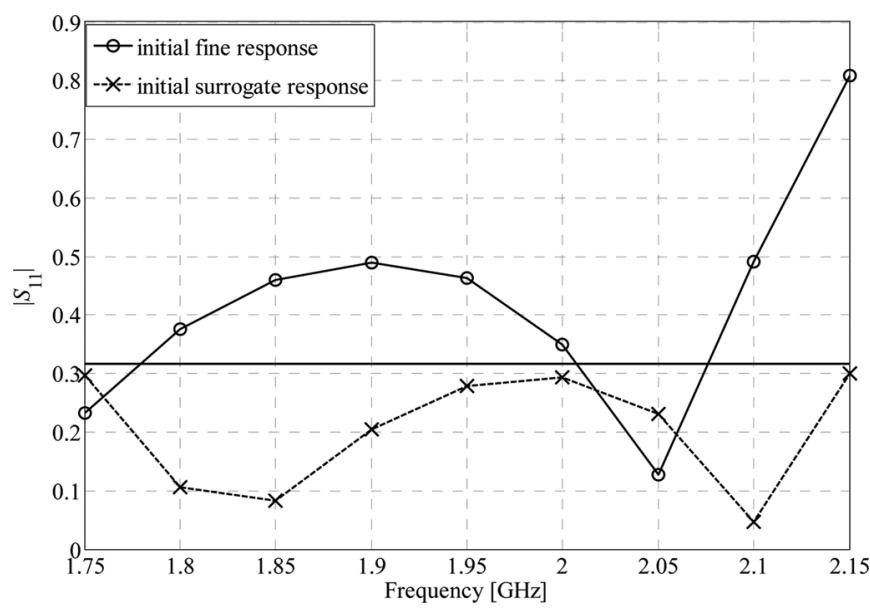

Fig. 5. Initial fine and surrogate responses corresponding to the coarse model optimal solution for the double annular ring antenna.

by local meshing. As shown in Fig. 4, the number of mesh lines along the three loops (thick lines) is topologically fixed at 5,10 , and 15 , respectively, regardless of the variation in the design parameter values. The fine and coarse models are summarized in Table I.

This problem has been solved using implicit and output SM. The relative permittivities of the two layers, $\varepsilon_{r 1}$ and $\varepsilon_{r 2}$, are used as preassigned parameters. The initial fine model, which takes its design-parameter values from the optimized coarse model (see Step 2 of the algorithm in Section II), exhibits a

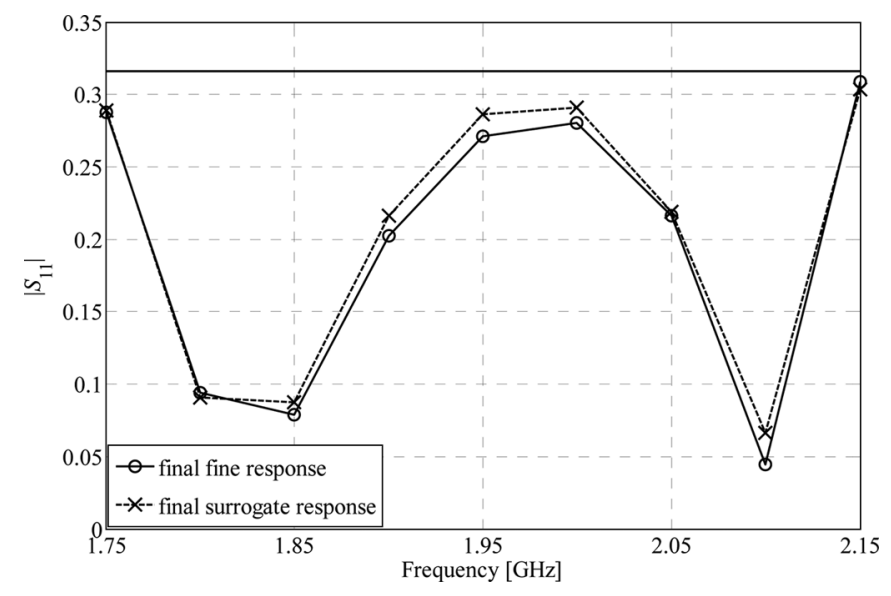

Fig. 6. Final fine and surrogate responses for the double annular ring antenna example.

TABLE II

INITIAL AND FinAl DESIGN OF THE DOUBLE ANNULAR RING ANTENNA

\begin{tabular}{ccc}
\hline \hline Design parameters & Initial design $(\mathrm{mm})$ & Final design $(\mathrm{mm})$ \\
\hline$a_{1}$ & 9.2277 & 10.6735 \\
$a_{2}$ & 8.7224 & 7.8088 \\
$b_{1}$ & 30.7230 & 28.4621 \\
$b_{2}$ & 34.1266 & 32.5043 \\
$\rho_{p}$ & 18.2107 & 19.6817 \\
\hline \hline
\end{tabular}

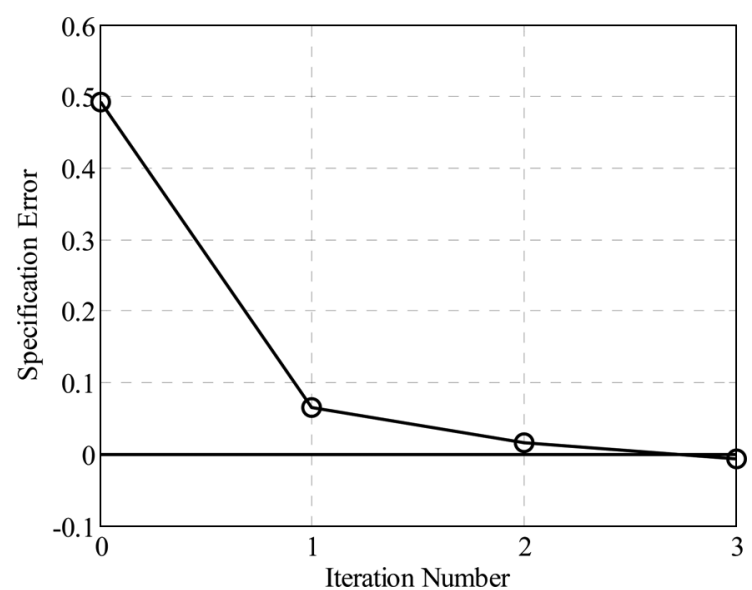

Fig. 7. Objective function value versus iteration number in the double annular ring antenna example.

poor response, shown in Fig. 5. In three iterations (four fine model simulations), the fine model is optimized and aligned with the surrogate very well. Both final responses are plotted in Fig. 6. The total time taken is $5 \mathrm{~h} 58 \mathrm{~min}$ (note that a single fine model simulation requires about $1 \mathrm{~h} 18 \mathrm{~min}$ ). Fig. 7 shows the reduction of the objective function versus the number of the iterations. Table II summarizes the initial and final designs.

Direct optimization of the fine model in this example was not attempted. With a simulation time of $1 \mathrm{~h}$ and $18 \mathrm{~min}$ per system analysis, direct optimization would require about a week, which is not acceptable.

We also note that Fig. 3 shows a general layout of the stackedring structure, which represents the relative sizes of the rings before the preliminary coarse-model optimization. In it, $a_{1}<$ $a_{2}$ and $b_{1}<b_{2}$. However, the coarse-model optimization as well as the subsequent SM optimization of the fine model resulted in a design where $a_{1}>a_{2}$ while $b_{1}$ remained smaller than $b_{2}$. 


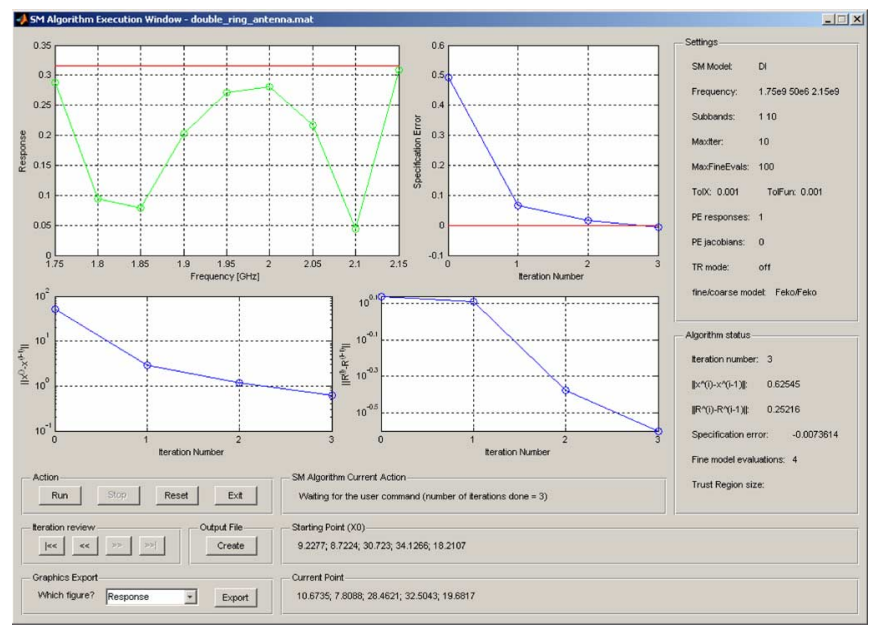

Fig. 8. Execution interface of SMF after the optimization procedure has stopped.

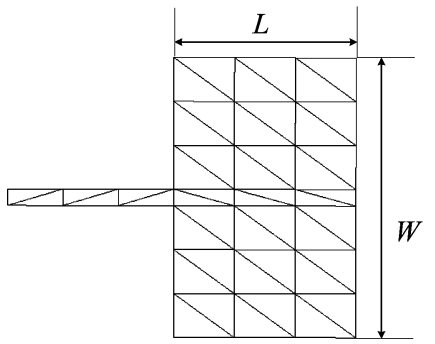

(a)

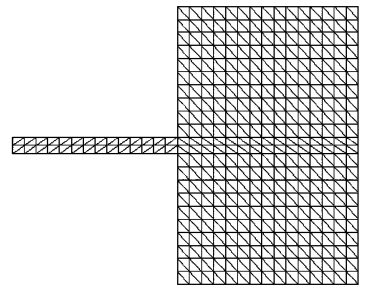

(b)
Fig. 9. Demonstration of the coarse model and the fine model. (a) The coarse model with three mesh lines along $L$ and seven mesh lines along $W$. (b) The fine model with global mesh density of 30 mesh lines per wavelength.

Finally, we show the execution interface of the SMF system at the final iteration of the SM algorithm in Fig. 8. Plots in the interface correspond to the algorithm status after the last iteration. The top left plot shows the fine model response and the design specifications; the top right plot shows the specification error versus iteration number; the bottom left and bottom right plots are convergence plots that show $\left\|\boldsymbol{x}^{(i+1)}-\boldsymbol{x}^{(i)}\right\|$ and $\left\|\boldsymbol{R}_{f}^{(i+1)}-\boldsymbol{R}_{f}^{(i)}\right\|$ versus iteration number, respectively.

\section{B. Patch Antenna}

The antenna is printed on a substrate with relative dielectric constant $\varepsilon_{r}=2.32$ and height $h=1.59 \mathrm{~mm}$. The design parameters are the patch length and width, i.e., $\boldsymbol{x}_{f}=[L W]^{T}$. The objective is to obtain $50 \Omega$ input impedance at $2 \mathrm{GHz}$. The objective function is $\left|Z_{i n}-50\right|$.

In the fine model, the global mesh density is 30 mesh lines per wavelength. In the coarse model, the mesh number and topology are fixed through local meshing. We choose three mesh lines along $L$ and seven along $W$. The meshes of the two models are shown in Fig. 9. We use implicit SM and output SM (SM plan I) where the selected preassigned SM parameter is $\boldsymbol{x}_{p}=\left[\varepsilon_{r}\right]$. In the parameter extraction, we match the complex $S_{11}$ instead of the input impedance.

The initial design point is the coarse model optimal solution $\boldsymbol{x}_{f}^{(0)}=\left[\begin{array}{ll}47.1285 & 100.470\end{array}\right]^{T} \mathrm{~mm}$. SM requires five iterations (six fine model simulations). Fig. 10 shows the reduction of the

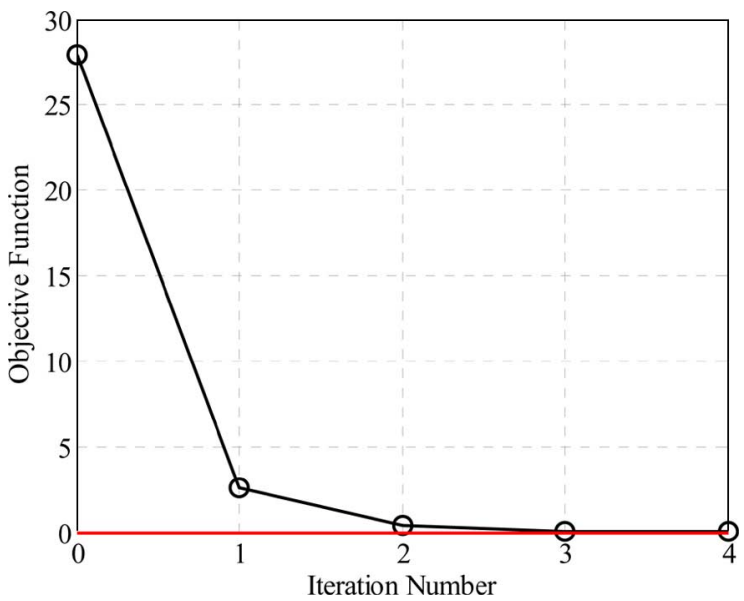

Fig. 10. Objective function value versus iteration number for the microstrip patch antenna example.

TABLE III

OPTIMIZATION RESUlTS FOR THE PATCH ANTENNA EXAMPLE

\begin{tabular}{ccccc}
\hline \hline Iteration & $\boldsymbol{x}_{f}(\mathrm{~mm})$ & $\varepsilon_{r}$ & $\Delta \boldsymbol{R}$ & $Z_{\text {in }}-50 \mid$ \\
\hline 0 & {$\left[\begin{array}{l}47.1285 \\
100.470\end{array}\right]$} & 2.3200 & 0.0000 & 27.941 \\
1 & {$\left[\begin{array}{c}46.77743 \\
99.6922\end{array}\right]$} & 2.3621 & $0.0082-0.0461 i$ & 2.5616 \\
2 & {$\left[\begin{array}{l}46.7268 \\
99.6960\end{array}\right]$} & 2.3589 & $0.0145+0.0056 i$ & 0.35956 \\
3 & {$\left[\begin{array}{l}46.7294 \\
99.6883\end{array}\right]$} & 2.3590 & $0.0118+0.0054 i$ & $2.4437 \times 10^{-2}$ \\
4 & {$\left[\begin{array}{l}46.7294 \\
99.6875\end{array}\right]$} & 2.3589 & $0.0115+0.0059 i$ & $1.1234 \times 10^{-2}$ \\
\hline \hline
\end{tabular}

objective function versus number of iterations. The final design is $\boldsymbol{x}_{f}^{*}=\boldsymbol{x}_{f}^{(4)}=[46.729499 .6875]^{T} \mathrm{~mm}$. Table III shows the evolution of the design parameters, the objective function, the preassigned parameter and the output SM parameter at each iteration. The computation time is $341 \mathrm{~s}$, compared with $2816 \mathrm{~s}$ for the direct fine model optimization.

An alternative way to solve this problem is through input and output SM (SM plan II). To save time in the parameter-extraction step, we extract only the variable vector $c$ in the input mapping ( $\boldsymbol{B}$ is fixed at $\boldsymbol{B}=\boldsymbol{I}$ ). The coarse model mesh is the same (100 mesh lines). The algorithm takes five iterations and $695 \mathrm{~s}$ to reach the specified error of $8.93 \times 10^{-3}$. As expected, it takes more time than the first SM option, because in the input SM, we tune two input SM variables in the parameter extraction rather than one as in the implicit SM.

Table IV summarizes the effect of the coarseness of the coarse model on the SM performance in the patch antenna example. The algorithm does not converge for 24 mesh elements (triangles). As the number of mesh elements increases, the function evaluation time increases while the SM iterations decrease. For SM plan I, we have the best SM performance in terms of total time cost for 100 mesh elements, which requires only $341 \mathrm{~s}$. For SM plan II, we have the best SM performance with 48 mesh elements, which requires $479 \mathrm{~s}$. 
TABLE IV

The Effect of Local Meshing on SM Performance for the Patch Antenna

\begin{tabular}{|c|c|c|c|c|c|c|c|}
\hline \multicolumn{3}{|c|}{ Local meshing in the coarse model } & \multicolumn{2}{c|}{ SM Plan I } & \multicolumn{2}{c|}{ SM Plan II } \\
\cline { 1 - 5 } Mesh number along & $\begin{array}{c}\text { Total mesh } \\
\text { number }\end{array}$ & $\begin{array}{c}\text { Function } \\
\text { evaluation time (s) }\end{array}$ & $\begin{array}{c}\text { Iteration } \\
\text { number }\end{array}$ & $\begin{array}{c}\text { Total time } \\
(\mathrm{s})\end{array}$ & $\begin{array}{c}\text { Iteration } \\
\text { number }\end{array}$ & $\begin{array}{c}\text { Total time } \\
(\mathrm{s})\end{array}$ \\
\hline 2 & $W$ & 24 & 0.109 & Not convergent & Not convergent \\
\hline 3 & 5 & 48 & 0.219 & 6 & 364 & 9 & 479 \\
\hline 5 & 9 & 100 & 0.438 & 4 & 341 & 5 & 695 \\
\hline 9 & 20 & 400 & 4.063 & 4 & 1604 & 3 & 2659 \\
\hline 11 & 22 & 528 & 8.375 & 3 & 2695 & 3 & 3220 \\
\hline \multicolumn{3}{|c|}{ Global meshing in the fine model } & \multicolumn{5}{c|}{ Direct optimization } \\
\hline
\end{tabular}

*The number of mesh lines and function evaluation time for the fine model is measured at the starting point $[L W]=\left[\begin{array}{ll}55 & 85\end{array}\right] \mathrm{mm}$.

\section{CONCLUSION}

We have presented an effective space-mapping technique for antenna optimization based on a coarse model, which exploits a coarse nonconvergent mesh of fixed topology. Both coarse and fine models are implemented in the same MoM solver. A separate coarse model is not required. In the double annular ring example, our SMF system provides an efficient way to address the finite ground size problem. We solve the optimal impedance of a patch antenna problem using two SM plans. The coarseness in the coarse model and its effect on the space mapping performance are discussed. The approach is applicable to the design of antenna and microwave devices aided by method-of-moments models.

\section{ACKNOWLEDGMENT}

The authors thank Dr. A. S. Mohamed and W. Yu of McMaster University for insightful comments.

\section{REFERENCES}

[1] P. Neittaanmäki, M. Rudnicki, and A. Savini, Inverse Problems and Optimal Design in Electricity and Magnetism. New York: Oxford Univ. Press, 1996.

[2] J. E. Dennis, Jr and V. Torczon "Managing approximation models in optimization," in Multidisciplinary Design Optimization, N. M. Alexandrov and M. Y. Hussaini, Eds. Philadelphia, PA: SIAM, 1997, pp. 330-374.

[3] N. M. Alexandrov, R. M. Lewis, C. R. Gumbert, L. L. Green, and P. A. Newman, "Approximation and model management in aerodynamic optimization with variable-fidelity models," AIAA J. Aircraft, vol. 38, no. 6, pp. 1093-1101, Nov.-Dec. 2001

[4] K. Madsen and J. Søndergaard, "Convergence of hybrid space mapping algorithms," Optimiz. Eng., vol. 5, no. 2, pp. 145-156, Jun. 2004.

[5] J. W. Bandler and K. Madsen, "Editorial-surrogate modelling and space mapping for engineering optimization," Optimiz. Eng., Special Issue on Surrogate Model. Space Mapping for Eng. Optimiz., vol. 2, no. 4, pp. 367-484, Dec. 2001.

[6] J. W. Bandler, R. M. Biernacki, S. H. Chen, P. A. Grobelny, and R. H. Hemmers, "Space mapping technique for electromagnetic optimization," IEEE Trans. Microw. Theory Tech., vol. 42, no. 12, pp. 2536-2544, Dec. 1994.

[7] J. W. Bandler, Q. S. Cheng, N. K. Nikolova, and M. A. Ismail, "Implicit space mapping optimization exploiting preassigned parameters," IEEE Trans. Microw. Theory Tech., vol. 52, no. 1, pp. 378-385, Jan. 2004.

[8] M. H. Bakr, J. W. Bandler, M. A. Ismail, J. E. Rayas-Sánchez, and Q. J. Zhang, "Neural space-mapping optimization for EM-based design," IEEE Trans. Microw. Theory Tech., vol. 48, no. 12, pp. 2307-2315, Dec. 2000

[9] M. A. Ismail, D. Smith, A. Panariello, Y. Wang, and M. Yu, "EMbased design of large-scale dielectric-resonator filters and multiplexers by space mapping," IEEE Trans. Microw. Theory Tech., vol. 52, no. 1, pp. 386-392, Jan. 2004

[10] K.-L. Wu, Y.-J. Zhao, J. Wang, and M. K. K. Cheng, "An effective dynamic coarse model for optimization design of LTCC RF circuits with aggressive space mapping," IEEE Trans. Microw. Theory Tech., vol. 52, no. 1, pp. 393-402, Jan. 2004.
[11] R. Garg, P. Bhartia, I. Bahl, and A. Ittipiboon, Microstrip Antenna Design Handbook. Norwood, MA: Artech House, 2001.

[12] R. A. Sainati, CAD of Microstrip Antennas for Wireless Applications. Norwood, MA: Artech House, 1996.

[13] C. A. Balanis, Antenna Theory, 3rd ed. Hoboken, NJ: Wiley, 2005.

[14] J. W. Bandler, S. Koziel, and K. Madsen, "Space mapping for engineering optimization," SIAG/Optimiz. Views-and-News, Special Issue on Surrogate/Derivative-Free Optimiz., vol. 17, no. 1, pp. 19-26, Mar. 2006.

[15] S. Koziel, J. W. Bandler, and K. Madsen, "Towards a rigorous formulation of the space mapping technique for engineering design," in Proc. ISCAS, Kobe, Japan, Jun. 2005, pp. 5605-5608.

[16] SMF, Bandler Corp.. Dundas, ON, Canada, L9H 5E7, 2006 [Online]. Available: http://www.bandler.com/SMF/

[17] "FEKO User's Manual" ver. Suite 4.2, [Online]. Available: http://www.feko.info, June 2004, EM Software Systems-S.A. (Pty) Ltd., 32 Techno Lane, Technopark, Stellenbosch, 7600, South Africa, In the USA: EM Software \& Systems (USA), Inc., 24 Research Dr., Hampton, VA 23666 USA.

[18] J. W. Bandler, Q. S. Cheng, D. Gebre-Mariam, K. Madsen, F. Pedersen, and J. Søndergaard, "EM-based surrogate modeling and design exploiting implicit, frequency and output space mappings," in IEEE MTT-S IMS Dig., Philadelphia, PA, Jun. 2003, pp. 1003-1006.

[19] S. Koziel, J. W. Bandler, and K. Madsen, "Space-mapping based interpolation for engineering optimization," IEEE Trans. Microw. Theory Tech., vol. 54, no. 6, pp. 2410-2421, Jun. 2006.

[20] “Advanced Design System 2003C, User's Manual," Agilent Technologies, Palo Alto, CA 94304 USA.

[21] "Mesh refinement," FEKO Quart., Dec. 2004

[22] D. M. Kokotoff, J. T. Aberle, and R. B. Waterhouse, "Rigorous analysis of probe-fed printed annular ring antennas," IEEE Trans. Antennas Propag., vol. 47, no. 2, pp. 384-388, Feb. 1999.

[23] F. Tavakkol-Hamedani, L. Shafai, and G. Z. Rafi, "The effects of substrate and ground plane size on the performance of finite rectangular microstrip antennas," in Proc. IEEE AP-S/URSI Int. Symp. Antennas Propag., San Antonio, TX, Jun. 2002, pp. 778-781.

[24] A. K. Bhattacharyya, "Effects of ground plane truncation on the impedance of a patch antenna," Inst. Elect. Eng. Proc.-H, vol. 138, no 6, pp. 560-564, Dec. 1991

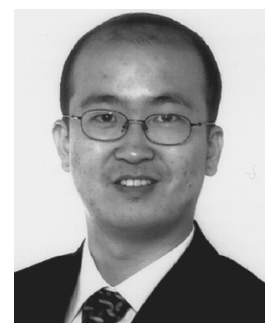

Jiang Zhu was born in Sichuan, China, in 1980. He received the B.Eng. degree in electrical engineering from Zhejiang University, Hangzhou, China, in 2003, and the M.A.Sc. degree in electrical engineering from McMaster University, Hamilton, ON, Canada, in 2006. He is currently working toward the $\mathrm{Ph} . \mathrm{D}$. degree at the University of Toronto, Toronto, ON, Canada.

In August 2003, he joined the Positioning and Wireless Technology Center, Nanyang Technological University, Singapore. From 2004 to 2006, he was a research assistant with the Computational Electromagnetics Research Laboratory and the Simulation Optimization Systems Research Laboratory, Department of Electrical and Computer Engineering, McMaster University. He joined the Electromagnetics Group, University of Toronto, in September 2006, where he is currently a Research Assistant. His research interests are electromagnetic metamaterials and their applications, electromagnetic modeling and optimization, microwave circuit, and antenna design. 


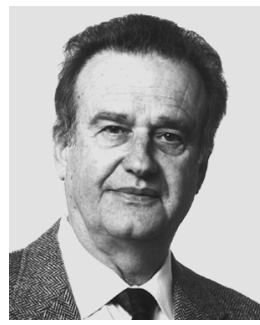

John W. Bandler (S'66-M'66-SM'74-F'78LF'06) was born in Jerusalem, on November 9, 1941. He studied at the Imperial College of Science and Technology, London, England, from 1960 to 1966. He received the B.Sc.(Eng.), Ph.D., and D.Sc.(Eng.) degrees from the University of London, in 1963, 1967, and 1976, respectively.

He joined Mullard Research Laboratories, Redhill, Surrey, England, in 1966. From 1967 to 1969, he was a Postdoctorate Fellow and Sessional Lecturer with the University of Manitoba, Winnipeg, Canada. He joined McMaster University, Hamilton, Canada, in 1969. He has served as Chairman of the Department of Electrical Engineering and Dean of the Faculty of Engineering. He is currently Professor Emeritus, directing research in the Simulation Optimization Systems Research Laboratory. He has published more than 390 papers from 1965 to 2006. He contributed to Modern Filter Theory and Design (New York: Wiley-Interscience, 1973) and to Analog Methods for Computer-aided Analysis and Diagnosis (New York: Marcel Dekker, 1988). Four of his papers have been reprinted in Computer-Aided Filter Design (New York: IEEE, 1973), one in each of Microwave Integrated Circuits (Norwood, MA: Artech House, 1975), Low-Noise Microwave Transistors and Amplifiers (New York: IEEE, 1981), Microwave Integrated Circuits, 2nd ed., (Norwood, MA: Artech House, 1985), Statistical Design of Integrated Circuits (New York: IEEE, 1987), and Analog Fault Diagnosis (New York: IEEE, 1987).

Dr. Bandler was President of Optimization Systems Associates Inc. (OSA), which he founded in 1983, until November 20, 1997, the date of acquisition of OSA by Hewlett-Packard Company (HP). OSA implemented a first-generation yield-driven microwave CAD capability for Raytheon in 1985, followed by further innovations in linear and nonlinear CAD technology for the Raytheon/Texas Instruments Joint Venture MIMIC Program. OSA introduced RoMPE in 1988, HarPE in 1989, OSA90 and OSA90/hope in 1991, Empipe in 1992, Empipe3D, and EmpipeExpress in 1996. OSA created the product empath in 1996 which was marketed by Sonnet Software, Inc., North Syracuse, NY. He is the President of Bandler Corporation, which he founded in 1997. He was an Associate Editor of the IEEE TRANSACTIONS ON MiCROWAVE THEORY AND TECHNIQUES (1969-1974), and has continued serving as a member of the Editorial Board. He was Guest Editor of the Special Issue of the IEEE TRANSACTIONS ON MICROWAVE THEORY AND TECHNiQues on COMPUTER-ORIENTED Microwave PRACTICES (1974) and Guest Coeditor of the Special Issue of the IEEE TRANSACTIONS ON MICROWAVE Theory AND TECHNIQUes ON PROCESS-ORIENTED MicrowaVe CAD AND MODELING (1992). He joined the Editorial Boards of the International Journal of Numerical Modelling in 1987, the International Journal of Microwave and Millimeterwave Computer-Aided Engineering in 1989, and Optimization and Engineering in 1998. He was Guest Editor, International Journal of Microwave and Millimeter-Wave Computer-Aided Engineering, Special Issue on Optimization-Oriented Microwave CAD (1997), and Guest Editor, the IEEE Transactions on MicrowaVE THEORY AND TECHNIQUES, SPECIAL ISSUE ON Automated Circuit Design Using Electromagnetic Simulators (1997). He was Guest Coeditor of Optimization and Engineering Special Issue on Surrogate Modelling and Space Mapping for Engineering Optimization (2001). He was Guest Coeditor of the IEEE TRANSACTIONS ON MICROWAVE THEORY AND TECHNIQUES, SPECIAL ISSUE ON ELECTROMAGNETICS-BASED OPTIMIZATION OF MicROWAVE COMPONENTS AND CiRCUITS (2004). He has served as Chair of the MTT-1 Technical Committee on Computer-Aided Design. He is a Fellow of the Canadian Academy of Engineering, a Fellow of the Royal Society of Canada, a Fellow of the Institution of Electrical Engineers (Great Britain), a Fellow of the Engineering Institute of Canada, a Member of the Association of Professional Engineers of the Province of Ontario (Canada), and a Member of the MIT Electromagnetics Academy. He received the Automatic Radio Frequency Techniques Group (ARFTG) Automated Measurements Career Award in 1994, and the IEEE MTT-S Microwave Application Award in 2004.

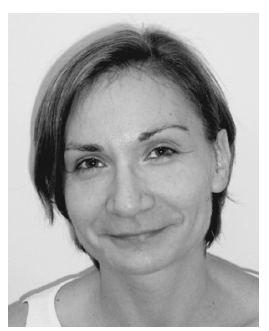

Natalia K. Nikolova (S'93-M'97-SM'05) received the Dipl. Eng. degree from the Technical University of Varna, Bulgaria, in 1989 and the Ph.D. degree from the University of Electro-Communications, Tokyo, Japan, in 1997.

From 1998 to 1999, she held a Postdoctoral Fellowship of the Natural Sciences and Engineering Research Council of Canada (NSERC), during which time she was initially with the Microwave and Electromagnetics Laboratory, DalTech, Dalhousie University, Halifax, Canada, and, later, for a year, with the Simulation Optimization Systems Research Laboratory, McMaster University, Hamilton, ON, Canada. In July 1999, she joined the Department of Electrical and Computer Engineering, McMaster University, where she is currently an Associate Professor. Her research interests include theoretical and computational electromagnetism, inverse electromagnetic problems and microwave imaging, as well as CAD methods for high-frequency structures and antennas.

Professor Nikolova was the recipient of a University Faculty Award of NSERC from 2000 to 2005. She is a member of Applied Computational Electromagnetics Society (ACES) and the International Union of Radio Science (URSI). She is a representative of Commission D in the Canadian National Committee of URSI and a member of the ACES Board of Directors.

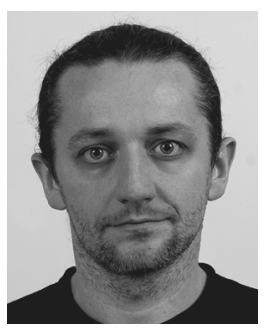

Slawomir Koziel (M'03) was born in Poland in 1970. He received the M.Sc. and Ph.D. (with honors) degrees in electronic engineering in 1995 and 2000, respectively, from the Gdansk University of Technology, Poland, and the M.Sc. degree in theoretical physics and in mathematics in 2000 and 2002, respectively, and the Ph.D. degree in mathematics (with honors) from the University of Gdansk, in 2003.

He is currently a Research Associate in the Simulation Optimization Systems Research Laboratory, Department of Electrical and Computer Engineering, McMaster University, Hamilton, ON, Canada. He has published more than 90 papers. His research interests include space mapping based optimization and modeling, circuit theory, analog signal processing, active filter design, evolutionary computation, and numerical analysis. 